\title{
COMPARISON OF PCA AND ICA BASED CLUTTER REDUCTION IN GPR SYSTEMS FOR ANTI- PERSONAL LANDMINE DETECTION
}

\author{
Brian Karlsen $^{(1)}$, Jan Larsen ${ }^{(2)}$, Helge B.D. Sørensen ${ }^{(1)}$ and Kaj B. Jakobsen ${ }^{(1)}$ \\ ${ }^{(1)}$ Ørsted•DTU, Technical University of Denmark \\ Ørsteds Plads, Building 348, DK-2800 Kongens Lyngby, Denmark \\ Web: http://www.oersted.dtu.dk, Email: brk,hbs,kbj@oersted.dtu.dk \\ ${ }^{(2)}$ Informatics and Mathematical Modelling, Technical University of Denmark \\ Richard Petersens Plads, Building 321, DK-2800 Kongens Lyngby, Denmark \\ Web: http://eivind.imm.dtu.dk, Email: jl@imm.dtu.dk
}

\begin{abstract}
This paper presents statistical signal processing approaches for clutter reduction in Stepped-Frequency Ground Penetrating Radar (SF-GPR) data. In particular, we suggest clutter/signal separation techniques based on principal and independent component analysis (PCA/ICA). The approaches are successfully evaluated and compared on real SF-GPR time-series. Field-test data are acquired using a monostatic S-band rectangular waveguide antenna.
\end{abstract}

\section{INTRODUCTION}

The development of techniques for automated detection of antipersonal landmines from sensor signal measurements is a significant problem. This paper focuses on improving signal-to-clutter ratio for detection systems based on ground penetrating radar (GPR) measurements. Clutter is characterized as signal components which are not directly correlated with primary scattering from mine objects. This comprises: measurement noise, disturbances from the antenna, inhomogeneities in the soil, scattering from rough surfaces, ground vegetation induced scattering, and to some extend multiple reflections. A number of recent clutter reduction approaches suggested in the literature cover: likelihood ratio testing [2], parametric system identification [3, 12, 15, 17], wavelet packet decomposition $[4,7]$, subspace techniques $[8,11$, $18,19]$, and simple mean scan subtraction [6].

We focus on unsupervised statistical based techniques for clutter reduction; in particular attenuation of surface disturbances. In Section 2 our previous suggested principal component analysis approach is revisited. Section 3 introduces a novel approach based on independent component analysis. Finally, Section 4 provides a comparative study on real GPR field test measurements.

JL is supported by the Danish Research Councils through the THOR Center for Neuroinformatics. BK acknowledges the Siemens Foundation for financial support. We thank Ole Nymann enthusiastic and steady support of our work in humanitarian mine detection. Furthermore, Staffan Abrahamson is acknowledged for collaboration on field data acquisition and Thomas Kolenda for valuable discussions on ICA.

\section{PRINCIPAL COMPONENT ANALYSIS CLUTTER REDUCTION}

Principal component techniques have previous been applied to GPR data analysis in [19] for detection of mines on preprocessed data using cross track-depth scans. In [18] clutter was reduced by reconstructing from the most significant eigenvectors, and [8] used a generalized singular values decomposition for separating noise and signal spaces. In [11] we took a different unsupervised approach where characteristics of the source signals (principal components) and associated eigenimages are used to determine the subspace for reconstruction.

Let $x_{i j}(t)$ denote the signal received at location $\mathrm{x}=(i-$ 1) $\mathrm{cm}, \mathrm{y}=(j-1) \mathrm{cm}$, where $i=1,2, \cdots, I$ and $j=1,2, \cdots, J$. Traditional clutter reduction [6] consists in subtracting the mean scan across the xy-plane, $\bar{x}_{i j}(t)=x_{i j}(t)-(I J)^{-1} \sum_{i, j} x_{i j}(t)$. This procedure removes the common signal across the xy-plane, which is mainly believed to originate from the very strong airto-ground reflection. The approach taken here is inspired by explorative analysis of functional neuroimages and multimedia data $[9,13]$. Define the $P \times N$ signal matrix: $\boldsymbol{X}=\left\{X_{p, t}\right\}, X_{p, t}=$ $\bar{x}_{i, j}(t)$, where the pixel index $p=i+(j-1) \cdot I \in[1 ; P]$, $P=I \cdot J . t \in[1 ; N]$ is the time index with $N$ being the total number of time samples. Column $t$ of the matrix then represent the $x y$-plane scan image at time $t$ reshaped into a vector, and the signal matrix represents the sequence of $x y$-plane images along the time or z-direction. Usually $P \gg N$ (in present experiments: $P=51^{2}=2601$ and $N=50$ ). Since the rank of $\boldsymbol{X}$ is at most $N$, the SVD reads

$$
\boldsymbol{X}=\boldsymbol{U} \boldsymbol{D} \boldsymbol{V}^{\top}=\sum_{i=1}^{N} \boldsymbol{u}_{i} D_{i, i} \boldsymbol{v}_{i}^{\top}, X_{p, t}=\sum_{i=1}^{N} U_{p, i} D_{i, i} V_{t, i}
$$

where the $P \times N$ matrix $\boldsymbol{U}=\left\{U_{p, i}\right\}=\left[\boldsymbol{u}_{1}, \boldsymbol{u}_{2}, \cdots, \boldsymbol{u}_{N}\right]$ and the $N \times N$ matrix $\boldsymbol{V}=\left\{V_{t, i}\right\}=\left[\boldsymbol{v}_{1}, \boldsymbol{v}_{2}, \cdots, \boldsymbol{v}_{N}\right]$ represent the orthonormal basis vectors, i.e., eigenvectors of the symmetric matrices $\boldsymbol{X} \boldsymbol{X}^{\top}$ and $\boldsymbol{X}^{\top} \boldsymbol{X}$, respectively. $\boldsymbol{D}=\left\{D_{i, i}\right\}$ is an $N \times N$ diagonal matrix of singular values ranked in decreasing order, as shown by $D_{i-1, i-1} \geq D_{i, i}, \forall i \in[2 ; N]$. The SVD identifies a set of uncorrelated time sequences, the principal components (PC's): $\boldsymbol{y}_{i}=D_{i, i} \boldsymbol{v}_{i}$, enumerated by the component index $i=1,2, \ldots, N$ and $\boldsymbol{y}_{i}=\left[y_{i}(1), \cdots, y_{i}(N)\right]^{\top}$. That is, we can 
write the observed signal matrix (image sequence) as a weighted sum of fixed eigenvectors (eigenimages) $\boldsymbol{u}_{i}$ that often lend themselves to direct interpretation: some will contain mostly clutter, whereas others mainly mine reflections.

Consider the projection onto the subspace spanned by $M$ selected PC's which mainly contain information about the mine object, i.e., $\boldsymbol{Y}=\widetilde{\boldsymbol{U}}^{\top} \boldsymbol{X}, \widetilde{\boldsymbol{U}}=\left[\boldsymbol{u}_{i_{1}}, \boldsymbol{u}_{i_{2}}, \cdots, \boldsymbol{u}_{i_{M}}\right]$, where $\boldsymbol{Y}$ is an $M \times N$ matrix. The selection can be done by inspecting the structure of the eigenimage or by the time course of $y_{i}(t)$. Ideally, if $y_{i}(t)=\delta\left(t-t_{0}\right)$ is a delta function, the structure of the eigenimage can be attributed to time $t_{0}$. The clutter is subsequently reduced by reconstructing $\boldsymbol{X}$ from the subspace, as given by $\widehat{\boldsymbol{X}}=\widetilde{\boldsymbol{U}} \boldsymbol{Y}$.

\section{INDEPENDENT COMPONENT ANALYSIS CLUTTER REDUCTION}

The spirit of the suggested method for independent component analysis (ICA) clutter reduction resembles that of the principal component based technique. The major difference is that the subspace formed by ICA is not orthogonal as in PCA. Moreover, the independent components (IC's), which are the counterparts of the PC's, are statistically independent. We thus expect the IC's to have a more distinct time localization.

Suppose that $\boldsymbol{X}$ first is projected to a subspace spanned by eigenvectors of non-zero eigenvalues, as we can not model from the null space [13]. Typically the dimension, $d$, of the signal subspace will be somewhat smaller than $N$. Let $\boldsymbol{U}$ be the $P \times d$ matrix of eigenvectors, and $\widetilde{\boldsymbol{X}}=\boldsymbol{U}^{\top} \boldsymbol{X}$ the projected signal matrix. The ICA problem is defined as: $\widetilde{\boldsymbol{X}}=\boldsymbol{A} \boldsymbol{S}$ where $\boldsymbol{A}$ is the $d \times M, M \leq d$, matrix of mixing coefficients and $\boldsymbol{S}$ is the $M \times N$ matrix of IC's - also referred to as source signals. That is, the original signal matrix is reconstructed as $\widehat{\boldsymbol{X}}=\boldsymbol{W} \boldsymbol{S}=$ $\sum_{i=1}^{M} \boldsymbol{w}_{i} \boldsymbol{s}_{i}^{\top}$, where $\boldsymbol{W}=\boldsymbol{U} \boldsymbol{A}$ is the matrix of eigenimages and $\boldsymbol{s}_{i}=\left[s_{i}(1), \cdots, s_{i}(N)\right]^{\top}$ is the $i$ 'th source signal. The literature provides a number of algorithms for estimating $\boldsymbol{A}$ and $\boldsymbol{S}^{1}$. Basically they can be divided into two families in which the first deploy higher (or lower) order moments of non-Gaussian sources, whereas the other family uses the time correlation of the source signals. In the present case we expect that the sources are both non-Gaussian and colored. We deploy a member from each family: the widely used Bell-Sejnowski [1] algorithm using natural gradient learning, and the Molgedey-Schuster algorithm [9, 16]. They are both able to estimate $\boldsymbol{A}$ and $\boldsymbol{S}$ up to a scaling factors and permutations of the source signals.

\section{EXPERIMENTS}

A comparison of the PCA and ICA methods for clutter reduction in GPR signals were performed on field-test Stepped-Frequency GPR data. The field-test data are collected using a monostatic Sband waveguide antenna operating in the frequency range $2.65-$ $3.95 \mathrm{GHz}$. The data were acquired using a HP8753C network analyzer. The bandwidth of the antenna determines the resolution which is approx. $11.5 \mathrm{~cm}$. After antenna deembedding [11] the signals were down-mixed to the base band in order to remove the carrier [6]. The deployed sampling frequency is $5.12 \mathrm{GHz}$, which

\footnotetext{
${ }^{1}$ For a resent review the reader is referred to [14].
}

corresponds to a free-space sampling of $2.93 \mathrm{~cm}$ in the depth direction, which is below the resolution set by the antenna bandwidth.

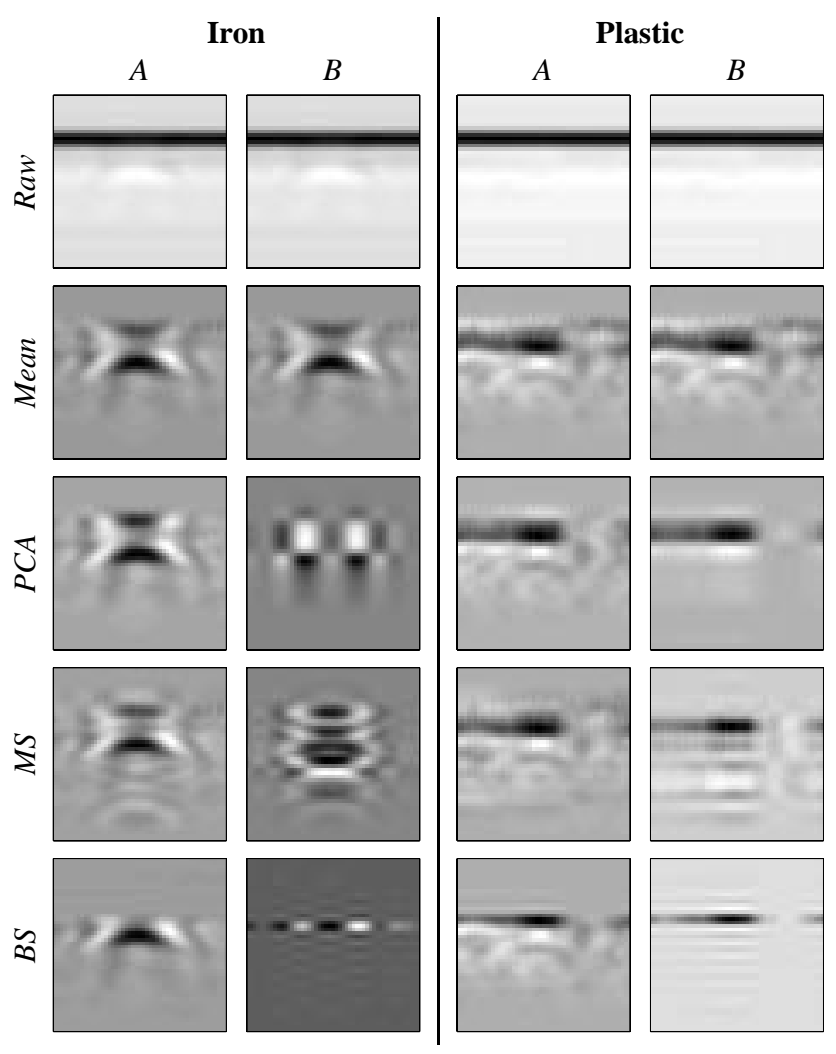

Fig. 1. Cross section (xt) images. The mine is located at the center in the $\mathrm{x}$-direction and at $t=16$ (2nd axis). The two left and right columns summarize results for iron and plastic mines, respectively. A columns correspond to reconstruction from components where only surface reflections are removed, and $B$ to reconstruction from the strongest mine, see Figure 2. The rows are: Raw data, Mean subtraction method, PCA, Molgedey-Schuster ICA $(M S)$, and Bell-Sejnowski ICA $(B S)$. Raw data shows only air-to-ground reflection whereas Mean method helps somewhat in reducing the strong surface reflection. $P C A$ seems to have a slight improvement over Mean, but $M S$ does not provide much improvement, and further seems to enhance multiple reflections. $B S$ on the other hand yields significant improvement, in particular when reconstructing from the strongest mine component only.

In a measurement area of $51 \mathrm{~cm} \times 51 \mathrm{~cm}$, M56 mine dummies $^{2}$ of iron and plastic (filled with bees wax) were buried in the center of the field in relatively dry sand $5 \mathrm{~cm}$ below the surface. The resulting signal matrices have $P=51^{2}=2601$ and $N=50$. The signal space dimension is $d=22$ for the iron mine and 17 for the plastic mine. Using a smaller area resulted in signal matrices which have too low signal space dimension. When using the BellSejnowski algorithm experiments show that appropriate learning rates are $10^{-4}$ and $10^{-3}$ for metal and plastic mine experiments, respectively. The lag value, $\tau$, for the Molgedey-Schuster algo-

\footnotetext{
${ }^{2}$ Dimensions are: diameter $5.4 \mathrm{~cm}$, and height $4 \mathrm{~cm}$.
} 
rithm turned out to be quite sensitive, but $\tau=1$ gave the best performance.

In Fig. 2 the eigenimages and associated PC's and IC's are depicted. ICA algorithms do not have any natural ordering. Since peak locations of the source signals determine the depth of scattering objects we choose to first rank according to peak locations occurring before the strong air-to-ground reflection at $t=16$. Next, the components are ordered wrt. to variance contribution ${ }^{3}$ in the reconstructed signal matrix [10], which for component $i$ is $\left|\boldsymbol{w}_{i}\right|^{2} \cdot \operatorname{Var}\left\{s_{i}(t)\right\}$

The eigenimages of the iron mine experiments show nearly all very strong mine signatures, however, more clearly pronounced for the ICA algorithms. It should be noticed that the added contribution from more components can display surface like texture. For instance, the contributions from components 1 and 4 of PCA will add to a more blurred overall contribution. The source signals of PCA and Molgedey-Schuster do not possess good time localiza$\operatorname{tion}^{4}$, thus associated eigenimages cannot be attributed to a particular depth. This also makes the selection of components for reconstruction somewhat unclear. On the other hand, the Bell-Sejnowski algorithm produces very peaked source signals. E.g., component 5 , which clearly peaks right after the surface reflection, also has a strong mine signature in its eigenimage. In addition, the width of the source peak is approximately 4 samples that corresponds to the resolution determined by the bandwidth of the antenna. Thus, source signals which have peak widths less than 4 samples do not make sense. The results for the plastic mine show that the mine signature is much less pronounced, i.e., signal-to-clutter ratio is low. Component 5 has a strong mine signature and is furthermore located at $t=18$, which is at the mine location. Recall that the mine has an extension of approx. $5 \mathrm{~cm}$ which is half the resolution set by the antenna bandwidth. The reconstructed cross-section images are shown in Figure 1.

\section{CONCLUSION}

This paper provided a comparative study of PCA and ICA algorithms for clutter reduction. In particular the Bell-Sejnowski ICA showed significant improvement over PCA and MolgedeySchuster ICA on real field GPR measurements. Future studies will focus on methods for automatic selection of subspace components and on convolutive ICA methods.

\section{REFERENCES}

[1] A. Bell \& T.J. Sejnowski, "An Information-Maximation Approach to Blind Separation and Blind Deconvelution," Neural Computation, vol. 7, pp. 1129-1159, 1995.

[2] H. Brunzell: "Clutter Reduction and Object Detection in Surface Penetrating Radar," in Proc. of IEE Radar'97, issue 449, 1997, pp. 688-691.

[3] J.W. Brooks, L. van Kempen \& H. Sahli: "Primary Study in Adaptive Clutter Reduction and Buried Minelike Target Enhancement from GPR Data," in Proc. of SPIE, AeroSense 2000: Detect. and Rem. Techn. for Mines and Minelike Targets $V$, vol. 4038, 2000, pp. 1183-1192.

\footnotetext{
${ }^{3}$ This measure is independent of the arbitrary scaling and permutation of the independent components.

${ }^{4}$ The Molgedey-Schuster algorithm most likely suffers from the fact that that the source signals are almost white.
}

[4] D. Carevic: "Clutter Reduction and Target Detection in Ground Penetrating Radar Data Using Wavelets," in Proc. of SPIE Conference on Detect. and Rem. Techn. for Mines and Minelike Targets IV, vol. 3710, 1999, pp. 973-97.

[5] P. Comon: "Independent Component Analysis: A New Concept," Signal Processing, vol. 36, pp. 287-314, 1994.

[6] D.J. Daniels: Surface Penetrating Radar, IEE, 1996.

[7] H. Deng \& H. Ling: "Clutter Reduction for Synthetic Aperture Radar Images Using Adaptive Wavelet Packet Transform," in Proc. of IEEE Int. Antennas and Propagation Society Symposium, vol. 3, 1999, pp. 1780-1783.

[8] A.H. Gynatilaka \& B.A. Baertlein: "A subspace decomposition technique to improve GPR imaging of anti-personnel mines," in Proc. of SPIE, AeroSense 2000: Detect. and Rem. Techn. for Mines and Minelike Targets V, vol. 4038, 2000, pp. 1008-1018.

[9] L.K. Hansen, J. Larsen \& T. Kolenda "On Independent Component Analysis for Multimedia Signals," in L. Guan, S.Y. Kung \& J. Larsen (eds.) Multimedia Image and Video Processing, CRC Press, Ch. 7, pp. 175-199, 2000.

[10] L.K. Hansen, J. Larsen \& T. Kolenda: "Blind Detection of Independent Dynamic Components," in Proc. IEEE ICASSP'2001, Salt Lake City, SAM-P8.10, vol. 5, 2001.

[11] B. Karlsen, J. Larsen, K.B. Jakobsen, H.B.D. Sørensen \& S. Abrahamson: "Antenna Characteristics and Air-Ground Interface Deembedding Methods for Stepped-Frequency Ground Penetrating Radar Measurements," in Proc. of SPIE, AeroSense 2000: Detect. and Rem. Techn. for Mines and Minelike Targets V, vol. 4038, 2000, pp. 1420-1430.

[12] L. van Kempen, H. Sahli, E. Nyssen \& J. Cornelis: "Signal Processing and Pattern Recognition Methods for Radar AP Mine Detection and Indentification," Detection of Abandoned Land Mines, no. 458, pp. 81-85, 1998.

[13] B. Lautrup, L.K. Hansen, I. Law, N. Mørch, C. Svarer \& S.C. Strother: "Massive weight sharing: A Cure for Extremely Ill-posed Problems," in H.J. Herman et al., (eds.) Supercomputing in Brain Research: From Tomography to Neural Networks, World Scientific Pub. Corp. 1995, pp. 137-148.

[14] T.W. Lee: Independent Component Analysis: Theory and Applications Kluwer Academic Publishers, ISBN: 0792382617, 1998.

[15] A. van der Merwe \& I.J. Gupta:A Novel Signal Processing Technique for Clutter Reduction in GPR Measurements of Small, Shallow Land Mines," IEEE Transactions on Geoscience and Remote Sensing vol. 38, no. 6 , Nov. 2000, pp. 2627-2637.

[16] L. Molgedey \& H. Schuster, "Separation of Independent Signals using Time-Delayed Correlations," Physical Review Letters, vol. 72, no. 23, pp. 3634-3637, 1994.

[17] J.L. Salvati, C.C. Chen \& J.T. Johnson: "Theoretical Study of a Surface Clutter Reduction Algorithm," in Proc. of 1998 IEEE International Geoscience and Remote Sensing, vol. 3, 1998, pp. 1460-1462.

[18] A.K. Shaw \& V. Bhatnagar: "Automatic Target Recognition Using Eigen-Templates," in Proc. of SPIE Conference on Algorithms for Synthetic Aperture Radar Imagery V, vol. 3370, 1998, pp. 448-459.

[19] S.H. Yu \& T.R. Witten: "Automatic Mine Detection based on Ground Penetratinig Radar," in Proc. of SPIE Conference on Detect. and Rem. Techn. for Mines and Minelike Targets IV, vol. 3710, 1999, pp. 961-972. 


\section{Iron Mine}
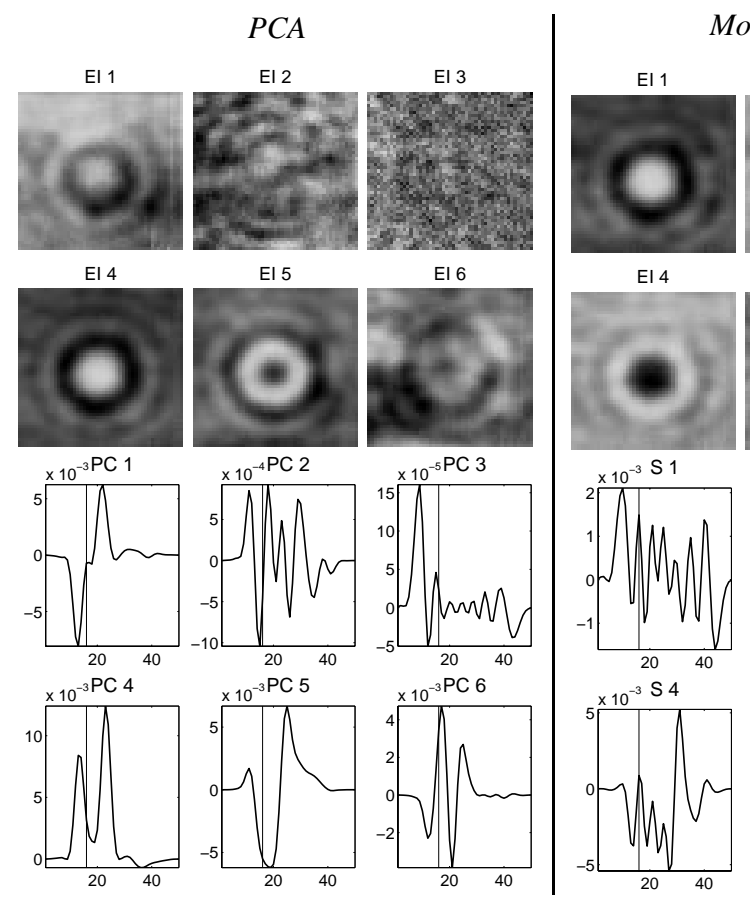

El 4
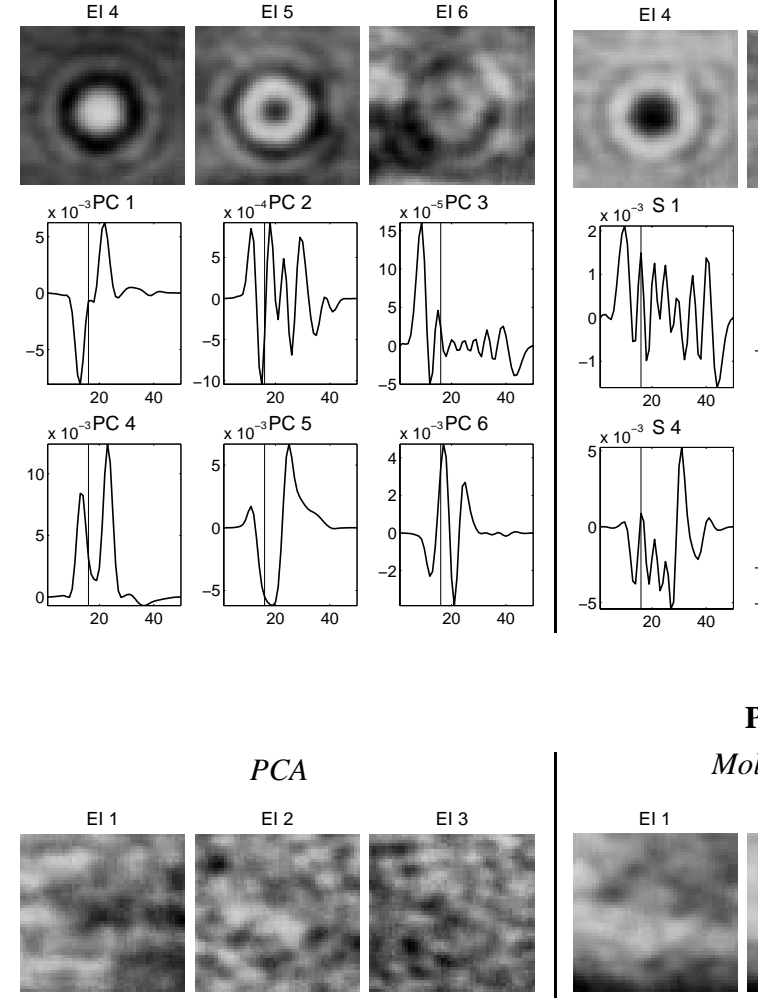

PCA

El 2

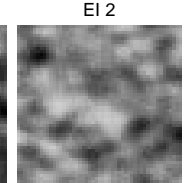

EI 5
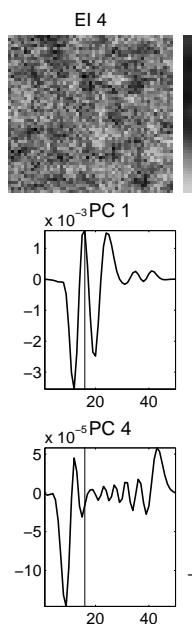

Molgedey-Schuster

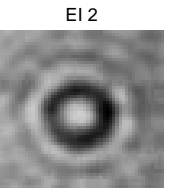

EI 5
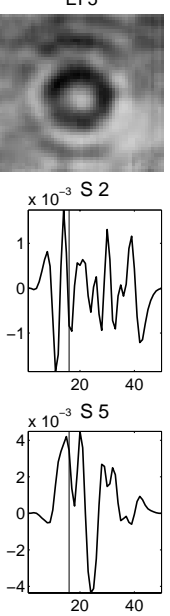

\section{Plastic Mine}

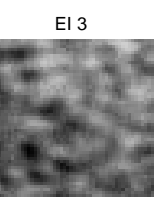

EI 6

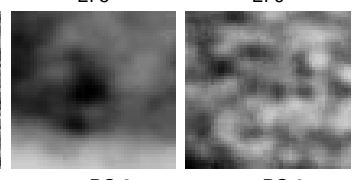

El 4

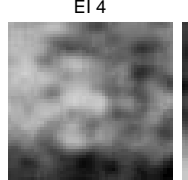

El 5
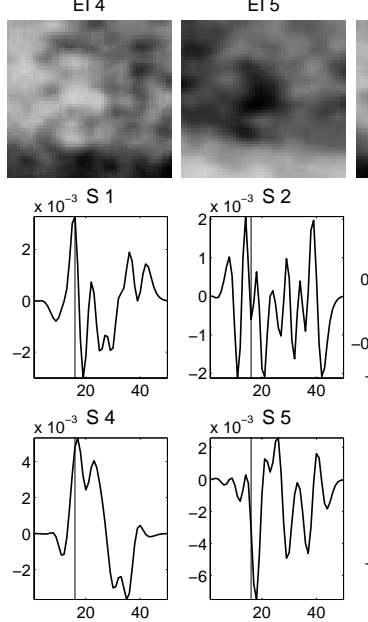

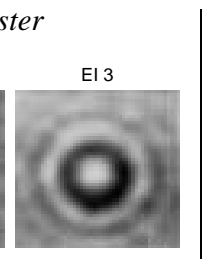

El 6
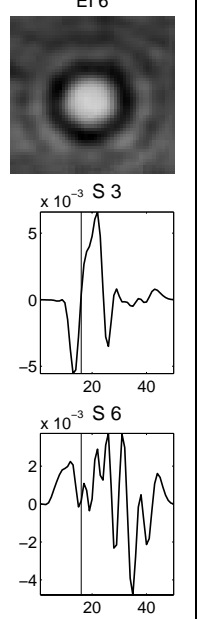

Bell-Sejnowski

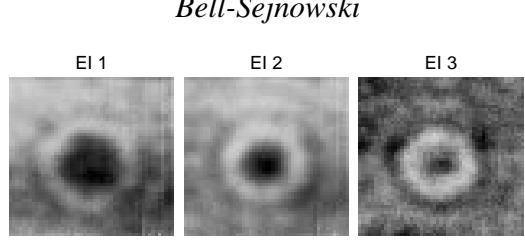

El 4
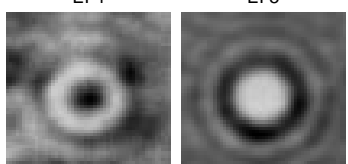

El 6
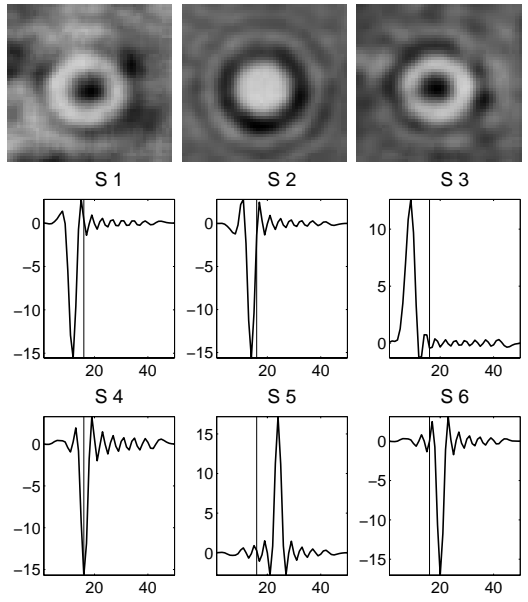

S 6

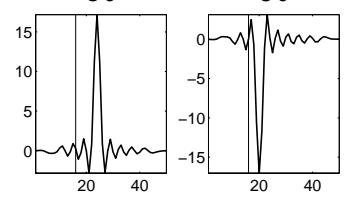

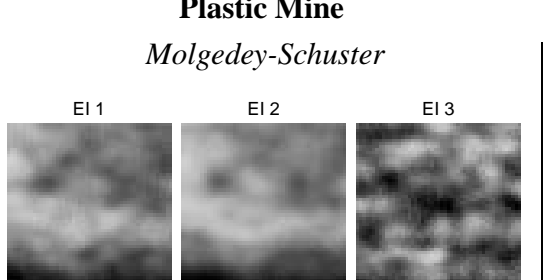

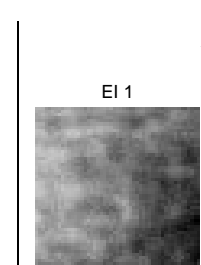

Bell-Sejnowski

El 6

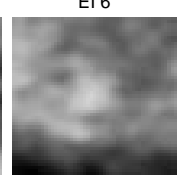

EI 4
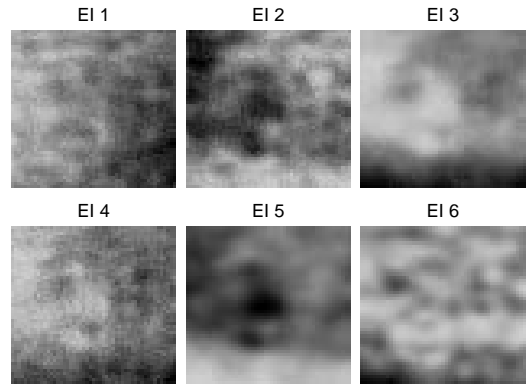

EI 6
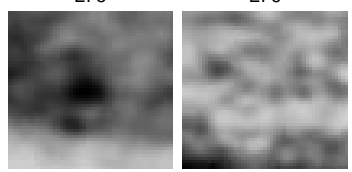

$\times 10^{-3} \mathrm{~S} 3$
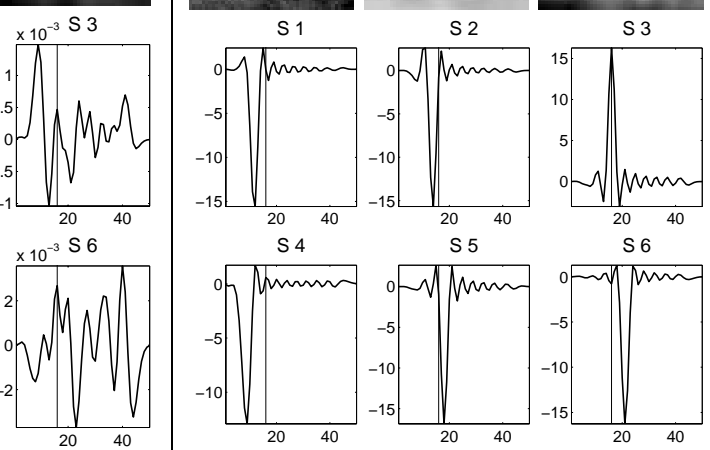

Fig. 2. Eigenimages and associated source signal, i.e., PC's or IC's. The vertical lines in the source signal pictures indicate the time corresponding to the position of the ground surface. Note that only the first 6 components are shown; the remaining source signals peak at later times and have smaller variance contributions. 